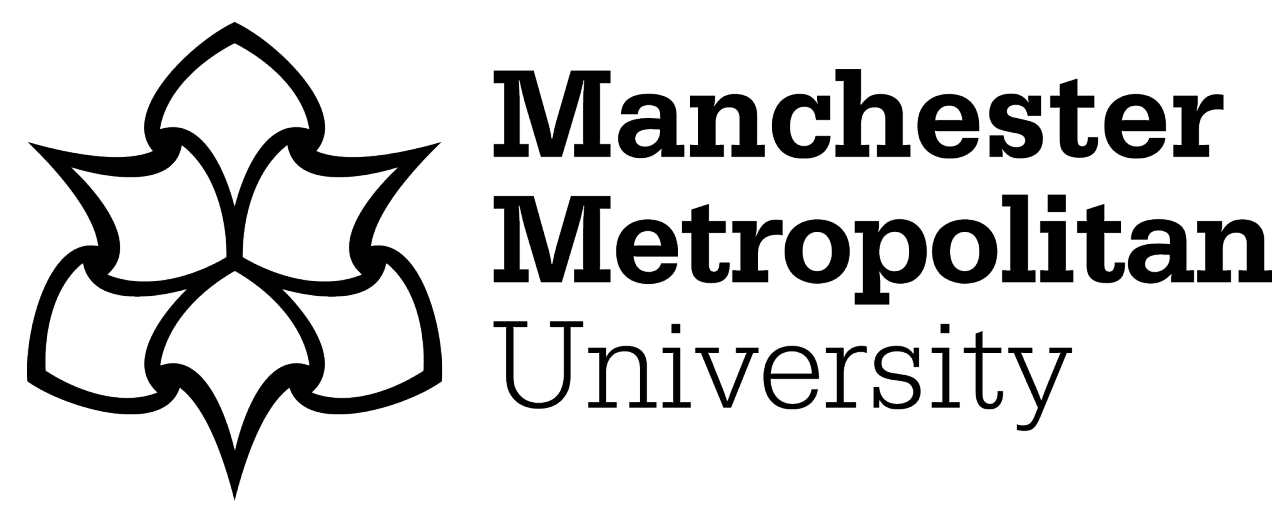

Henry, John ORCID logoORCID: https://orcid.org/0000-0003-3674-8208, Tang, Stephen, Mukhopadhyay, Subhas and Yap, Moi Hoon (2021) A Randomised Control Trial for measuring Student Engagement through the Internet of Things and Serious Games. Internet of Things, 13. p. 100332. ISSN 2542-6605

Downloaded from: https://e-space.mmu.ac.uk/626882/

Version: Accepted Version

Publisher: Elsevier BV

DOI: https://doi.org/10.1016/j.iot.2020.100332

Usage rights: Creative Commons: Attribution-Noncommercial-No Derivative Works 4.0

Please cite the published version 


\title{
A Randomised Control Trial for measuring Student Engagement through the Internet of Things and Serious Games
}

\author{
John Henry ${ }^{\mathrm{a}, 1, *}$, Stephen Tang ${ }^{\mathrm{b}}$, Subhas Mukhopadhyay ${ }^{\mathrm{c}}$, Moi Hoon Yap ${ }^{\mathrm{a}, 1}$ \\ ${ }^{a}$ Department of Computing and Mathematics, Faculty of Science and Engineering, \\ Manchester Metropolitan University, John Dalton Building Chester Street, Manchester, M1 \\ $5 G D U K$ \\ ${ }^{b}$ Tang Inclusion Ltd, United Kingdom \\ ${ }^{c}$ School of Engineering, Faculty of Science and Engineering, Macquarie University, Waterloo \\ Rd after, Coolinga St, Macquarie Park NSW 2113, Australia
}

\begin{abstract}
The combination of the Internet of Things (IoT) with Serious Games is an academic domain of research increasing in popularity. Combining Serious Games with the interconnected hardware and middleware driven ecosystem of IoT develops data-driven games that source data from the local or extended physical environment to progress in the virtual environment of gaming, better-informing players through the game experience. The following paper presents the evaluation of a software framework defined for combining Serious Games and IoT, achieved using a semester-long experiment with a randomised control trial. The study aimed to produce an effective method of measuring student engagement using sensor-based information and a game environment but not improve the level of student engagement with their related academic programme. The results of the empirical experiment provide perceived evidences in the measure of engagement when compared to academic performance. The experiment results are not statistically significant due to the small sample size and the empirical nature of the work. The findings of this paper validate the effectiveness of a software framework for combining Serious Games and IoT and encourages new real-world applications of Smart Serious Games that include healthcare, education, simulation and others.
\end{abstract}

Keywords: Randomised Control Trial, Development Framework, Internet of Things, Serious Games

\footnotetext{
*Corresponding Author Email Address: john.henry@mmu.ac.uk (John Henry)

Email addresses: mr.stephentanggmail.com (Stephen Tang), subhas.mukhopadhyay@mq.edu.au (Subhas Mukhopadhyay), m.yap@mmu.ac.uk (Moi Hoon Yap)
} 


\section{Introduction}

The Internet of Things (IoT) details an ecosystem comprising of interconnected devices, middleware and users that operate in Smart Environments $[1,2]$. The term Serious Games describes computer games or video games built for non-entertainment domains [3, 4], with 'serious' goals including education and positive behavioural modification with emphasis on reaching an end-goal through user interaction. IoT and in particular interconnected sensor devices and data from the physical environment present promise in evolving Games Serious Games and Gamification. The presented work focuses on the relationship between IoT and Serious Games.

Favorskaya et al. [5] define the combination of the IoT ecosystem with Serious Games as Smart Serious Games (SSGs). Further to providing a definition, they detail the advantages of SSGs. Summarising on their views, SSGs are tools that can solve real-world problems in any domain where game technology can assist. By nature, these games are data-driven, or event-driven. In either situation, data forms the driving force of development rather than game-play experience alone. The use of IoT with gaming technologies complements advances in augmented reality and virtual reality gaming.

Thus far, the related research project developed an application neutral and modular framework for combining IoT and Serious Games [6]; however, the framework lacked validation due to its preliminary nature, limiting its effectiveness. Application neutral describes a framework that disregards the specific application or research project, but can be utilised for any application that combines IoT with Serious Games. The study presents a novel software development framework complete with data analysis following an empirical study and a novel measure of student engagement using the Internet of Things to monitor attendance and game technology to record questionnaire responses. To our knowledge, no existing literature provides a validated application neutral software framework that outlines the intercommunication of IoT and Serious Games. This paper fills this void in the scientific knowledge-base by attempting to validate a software development framework by creating a solution that integrates the Internet of Things and Serious Games to measure student engagement. The successful development of a Serious Game that integrates with IoT will determine if the proposed framework is effective. We hypothesize that if the produced application can measure as effectively as conventional methods of student engagement, the framework can be considered as validated. Our research sets a foundation for future research in health care, rehabilitation, education and immersive games that utilise IoT and Serious Games.

The validation of the defined software framework occurred through a semesterlong, Randomised Control Trial (RCT) experiment. The experiment produced two control groups for measuring Student Engagement, one through self-reflection instruments alone, the second through a game that utilises data obtained from the local and extended environment using IoT, to overlay a measure of behavioural engagement onto self-reflection instruments.

Measuring engagement has benefits that extends beyond education. The en- 
gagement theory relates to a vast setting type, including employee engagement and others. Research into the impact of engagement on employees identifies a correlation between performance and engagement $[7,8]$. This fact remains true when investigating the correlation between Student Engagement and student performance [9]. Furthermore, a measure of Student Engagement indicates student dropout, as research proves an affiliation between the two [10, 11]. Therefore, correlating against student academic performance validates the obtained measures of Student Engagement. Section V provides further information on data analysis.

The following section presents the background research surrounding the combination of Serious Games and IoT, with emphasis on the latest research related to the topic. Section III presents the updated modular framework for Serious Games and the IoT, and details the development of an SSG named SEA, with emphasis on how multi-element systems create a data-driven virtual gaming environment that reacts to the physical world. Section IV presents the data analysis and conclusions of data obtained from a semester-long RCT experiment. Finally, Section V concludes on the key points and findings of this paper and suggests future directions of research.

\section{Related Work}

IoT is expanding, with research investigating Fog Computing [12, 13] and development of the Industrial Internet of Things (IIoT) also known as Industry 4.0 [14]. IoT is also making an impact on Games with more games attempting to source data from the physical environment to affect gameplay in the virtual environment. The combination of Serious Games with IoT advances Serious Games, Gamification and Edutainment. The following section presents the latest and key literature in the domain. Existing research into this academic field has proposed applications or application-driven frameworks that are not adaptable in nature $[15,16,17]$, therefore limiting the scope of application to a bespoke application. Our software framework addresses the gap in existing scientific literature by presenting a validated, modular software framework that is not bespoke to a single application.

\subsection{The Internet of Things and Serious Games}

Literature in SSGs is limited in comparison to Gamification or Serious Games as the domain and term was defined in 2015, with some research projects beginning to include the term as future work $[18,19]$. Research into SSGs is vital, as IoT delivers interconnected sensors for better data acquisition and a pervasive experience, elements that create quantitative results with less intrusive methods of obtaining them. In addition, player behaviour can be analysed in correlation to new sets of data, something previously not possible.

In a truly interconnected IoT ecosystem, Serious Games could harvest and analyse data from players' physical worlds or smart cities [20] and present it to the user, to provide better player behaviour insights. Harnessing player 
behaviour data, allows game developers to improve game-play, provides more robust conclusions on research surrounding games and Serious Games, and aids the industry to tailor in-game content to match player satisfaction, amongst others. The following sections highlight the integration of IoT with Serious Games through a validated software framework and investigate the benefits of data analytics on Student Engagement measurement.

Thus far, the published literature by Henry et al. [6] presented a preliminary version of the software framework for combining IoT and Serious Games, with no validation, as it was not possible at the time their literature was published. This paper addresses the lack of validation and produces the final iteration of the software framework.

\subsection{The Internet of Things and Edutainment}

Applications that utilise Edutainment and the IoT are appearing in health care [21], education in schools [22] and museums [23]. Additionally, research in the domain has investigated the use of embedded systems [24]. Our validated

software framework provides an effective foundation for future applications of Edutainment that combine with IoT.

In detail, Vicini et al. [21] presented educating young patients in a hospital environment through IoT and edutainment amongst other domains. Their research highlights the versatile benefits of combining game technology and IoT. Our validated framework complements the research of Vicini et al., proposes a new perspective of application through IoT, and blueprints how to achieve it.

Research into IoT and edutainment within educational settings includes the use of toys in early learning, to engage students through trans-media play. Heljakka and Ihamaki [22] took the definition further by presenting the term IoToys for IoT interconnected toys. Regardless of this definition, the project utilises IoT and edutainment to promote education. Our validated framework allows future researchers to further the research of Heljakka and Ihamaki with a practical illustration of how IoT and its ecosystem must integrate into Game applications.

Lien [23] presented research into a mobile focused edutainment system, applied in museums. Her research proposed an embedded approach of edutainment by producing games that promote further learning but did not produce a framework for the development of such systems. Our software framework provides a solution to the void in current scientific literature surrounding the integration of Serious Games and Edutainment with IoT. There is no available literature that produces an effective software framework for this combination, following experimentation and data analysis.

Kranz et al. [24] presented alongside their research into embedded interaction an edutainment application with micro-controllers for input. The project proved that these applications were effective for education, particularly for short memory exercises. Our research furthers such applications by introducing the validated software framework required to develop this type of game, illustrating how it expands to include extrinsic sensors that embed directly into the game. 


\subsection{The Internet of Things and Gamification}

There is limited research that explores the combination of Gamification with IoT. Research into Big Data, crowd-sensing and Gamification [25] and utilising sensors with Gamification for several applications [26, 27] provide promise for the domain of Gamification and IoT. Our validated software framework contributes to the void in the academic field as it remains applicable to combinations of Gamification and IoT.

Research by Crowley et al. [26], investigated the use of citizen sensing and gamification, for reporting issues in players' local environment. Their research outlines a framework for this integration and presents an example of relevant research into combining the physical world with game technology. This paper presents a validated software framework that extends the research of Crowley et al. and any other projects related to combining IoT with Gamification.

Gamification and IoT are predicted to affect healthcare. The literature survey by Islam et al. [27] into IoT and healthcare, suggests wearable devices as an avenue of integrating Gamification into IoT for patient engagement and public health information. These possibilities are valid but can extend to rehabilitation, mental health and physical health in athletes, amongst others. Therefore, a validated software framework detailing the development process of combining Serious Games or Gamification with IoT advances the research field into making the proposed applications a reality.

The research by Swan [28] into applications of sensor technologies highlighted gamification as an area of high potential as it allows sensor-based data to provide users with recommendations, creating a smart solution. Our research extends this idea by producing the validated software framework required for combining these technologies and progresses the scientific field into new applications.

\section{A Development Framework for The Internet of Things and Serious Games}

Henry et al [6] outlined the software architecture and presented a preliminary version of the software framework that enables the development of any application related to SSGs. The final iteration of the software framework presented in Fig.1 improves on the preliminary framework by:

- Validating the framework based on an RCT experiment.

- Correcting the data flow between models and layers.

- Highlighting the points of distinction between each segment.

The software framework is valid for data- driven applications as it details the intercommunication of a system that relies on time and place. Any application that requires IoT for such a scenario benefits from the software framework, even solutions that do not embed game technologies. Furthermore, the software framework is adaptable as it would maintain its significance for IoT research projects into RFID, such as Yang et al. [29]. 


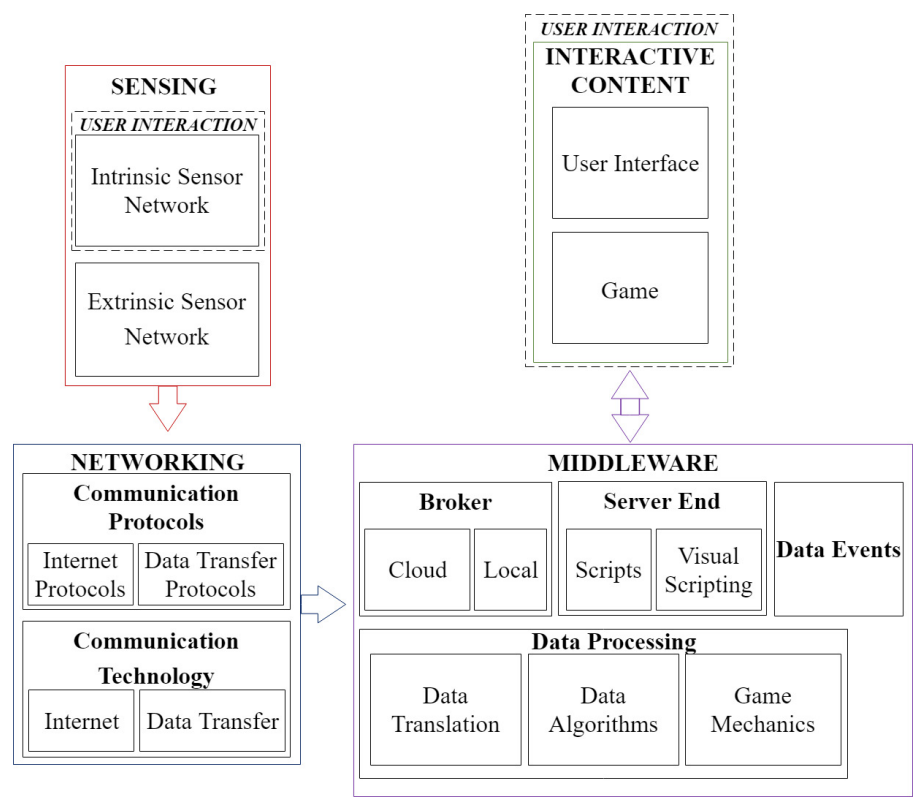

Figure 1: The proposed development framework for combining IoT and Serious Games, with improved readability.

We present the developed SSG, namely Student Engagement Application (SEA), that overlays conventional Student Engagement measures [10, 30, 31, 9, 32] using a hybrid wireless sensor network to measure attendance and punctuality as a measure of Student Engagement.

The following details the relationship and presence of IoT in the validated software framework, and explains the process of combining data into a game or gamified application, sourced from the broader environment through extrinsic sensor networks, and the local environment from intrinsic sensor networks.

\subsection{Sensing}

SEA required multiple systems to intercommunicate before measuring Student Engagement. Developing for IoT involves an understanding of networking, middleware and scripting languages. Additionally, development must evaluate scalability and network load, following the topology requirements.

\subsubsection{Intrinsic Sensor Networks}

SEA requires data from the local physical environment to detect presence at a location and time. Network nodes consisting of microcomputers developed an intrinsic senor network of remote autonomous nodes with interchangeable locations. Microcomputers represented nodes due to their low power, portability and affordability. The software framework extends past microcomputers as nodes; however, they include onboard Bluetooth and Wi-Fi capabilities, eliminating the need for purchasing and configuring additional adapters. 
The nodes run on a variation of the Linux operating system, which provides all the extensive support of Linux, with minimum bloatware. The automated attendance system utilises shell scripts for execution at start-up. The nodes serve two functionalities; discover connected devices on a set interval of two minutes and listen for a request to change the current location of the node. Figure 2 illustrates the essential programming structure for nodes.
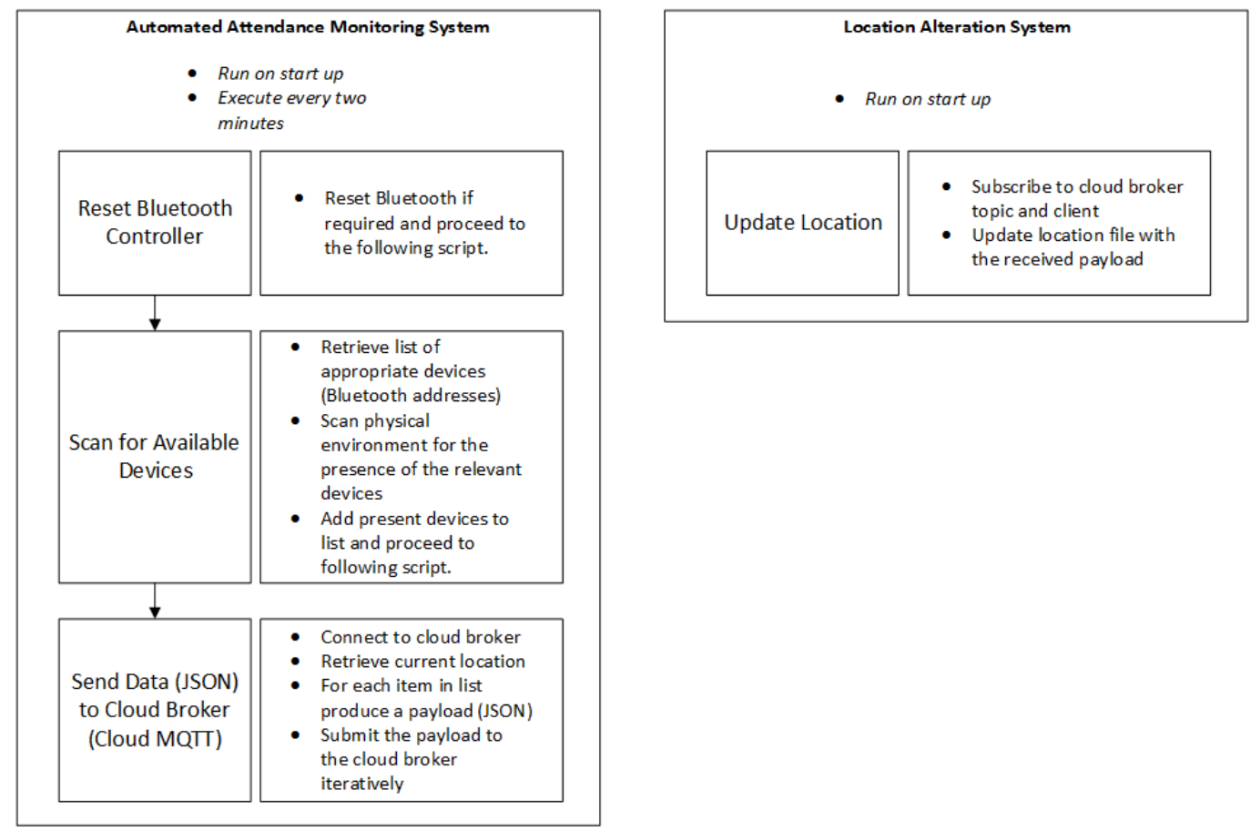

Figure 2: System component diagram and flow of the Internet of Things attendance system.

\subsubsection{Extrinsic Sensor Network}

Extrinsic networks relate to the middleware section; however, they differ in their nature and flow style. In SEA, extrinsic sensor networks feed data to NodeRED through API, which determines when an API call should be triggered. SEA utilises data from two API sources; weather data from the Met Office, and traffic data from HERE Technologies.

The system operates on a one to one basis, where one script checks the timetable for each related programme of study. A one to one relationship provides scalability and allows concurrent processes to run without the need for a complicated programming solution. Other API sources may be added following similar flows. SEA generates a list of the weather and traffic conditions for each day and time a student is late for, or absent from, a lecture or practical session. Continuing, the SSG detects which state re-occurred most commonly and informs the student in-game. The information changes weekly. By presenting the 
information above to the student, they may uncover patterns regarding their behaviour, which they may choose to change.

Extrinsic sensor networks feed data to Node-RED through API. However, it is Node-RED that determines when an API call should be triggered. Figure 3 the flow for obtaining data from two API sources; weather data from the Met Office, and traffic data from HERE. The top left node schedules an API call to run every weekday at one in the morning. This allows the system to retrieve the full twenty-four-hour forecast for the day. If an error occurs it formats the payload appropriately, then emails the author for debugging purposes.

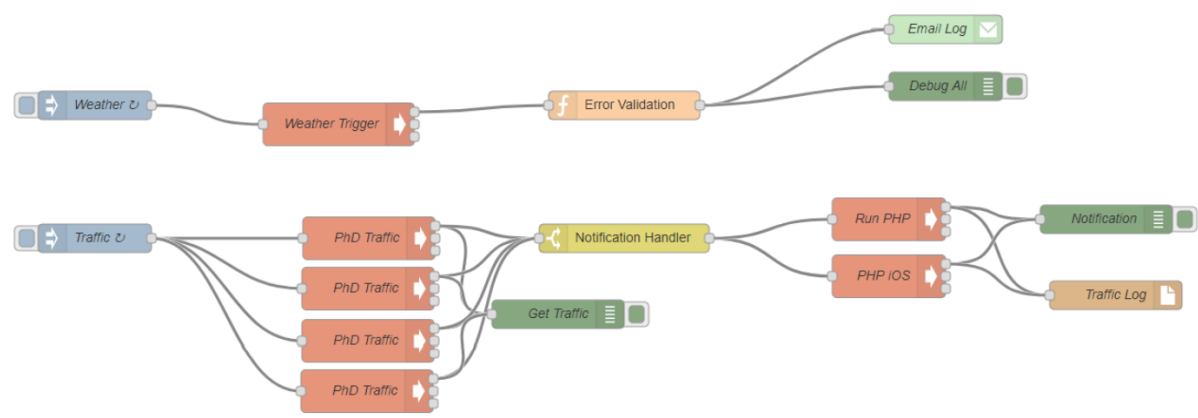

Figure 3: Node-Red visual script of the API system that sources data from wider environment.

The traffic API runs on a five-minute interval, every weekday, from eight in the morning until six in the evening. This interval allows the system to cover all timetabled sessions for each relevant programme of study, and to obtain the traffic an hour before the start of a session. Figure 3 illustrates four trigger nodes for executing four different hosted scripts.

The system has been developed on a one to one basis, where one script checks the timetable for each related programme of study. This provides scalability and allows concurrent processes to run without the need for a complicated programming solution. After the data is stored, the traffic congestion is analysed for each student on the programme. If the congestion time is higher than the average in comparison to a student being late or absent, a notification alerts them to current traffic conditions. Other API sources may be added following similar flows.

Extrinsic networks integrate into the system and provide useful information to the student. The inclusion of other sources of data could provide new correlations and insights, and thus form part of the future work recommendations.

\subsection{Networking}

The networking section of the software framework considers the network protocols and technologies that are mandatory when developing an SSG. SEA requires portability, low power and network accessibility to operate in a university campus, obtain a presence in a specific location and time, and allow players 
to access their game data from any location with an Internet network. As such, SEA comprises intrinsic sensor networks that utilise BLE to detect the presence of a mobile device. BLE meets the low power requirement while maintaining a sufficient range of discovery. The information from a node utilises Wi-Fi to send data packets to a cloud message broker, through to a hosted server.

SEA features asynchronous network transmission when sending information through the intrinsic sensor network and when obtaining information as a client in the middleware and interactive content sections. The asynchronous communication allows the monitoring of simultaneous classes and complies with network accessibility.

\subsection{Middleware}

The middleware module of the software framework comprises of the inter-

communication between sensor networks and game technology and consists of three key elements; the cloud broker, a programming tool, and hosted scripts for data validation and data storage. SEA utilises Cloud MQTT for the cloud broker, Node-RED for the programming tool, and a hosted area accompanied by a hosted database.

Cloud MQTT provides free cloud brokerage, limited to 10 connections and a 10Kbit/s transfer rate. SEA caters for the restrictions set by the cloud broker by minimising the payload to one or two elements, adhering to the limited transfer rate. Minimising the payload presents further benefits such as reduced network demand and latency. The cloud broker holds twelve topics, two per active node. The choice of cloud broker does not affect the composition of the software framework, nor does it affect the results of the measure on Student Engagement.

All messages sent to and from the cloud broker interacted with the NodeRED, a programming tool developed by IBM and based on Node.JS, which allows for runtime execution. Node-RED utilises visual scripting to reduce development time, labelled as flows. This project incorporated Node-RED as it offers a free solution to developing a scalable Internet of Things system, with minimum development time. Node-RED runs locally but receives and transmits messages to the cloud broker, and triggers hosted scripts. For the purpose of this case study, each node had a separate palette, detailed in Figure 4. The top half of Figure 4 illustrates the visual code developed for receiving the Bluetooth Address and location of a present node. The sequence flows from left to right.

The initial node connects to the cloud broker and listens for messages from a specific user and topic, in this example the first wireless network node. Upon receiving a message, the brown nodes generate local and hosted copies of the data for debugging purposes, and to be read by the hosted script responsible for data validation and storage. As seen in Figure 4, a node delays the execution of the hosted PHP script by two seconds. The delay provides enough time to overwrite the relative file that stores the respective Bluetooth Address and location iteratively. As an alternative, it is possible to feed information directly to PHP through the cloud broker. The use of a local file does not impede the measure of Student Engagement obtained from the system. The final key 
node in the getLocation section triggers the hosted PHP script, responsible for validating presence against a student's timetable, and inserting data into the database, whilst preventing duplicate entries.

The setLocation section contains a node for receiving and publishing messages to the cloud broker. The switch node to the right determines the flow of events. If the payload message contains the words "Location updated" it will send an email to the academic staff responsible for the remote network node, otherwise it will do nothing, as it is the network node's responsibility to set the location. A payload message will only state the aforementioned phrase when the network node updates the location file and transmits the message to the same account and topic through the cloud broker. This feature provides academic staff with feedback when a location is changed through the academic portal, detailed in Section 6.3.

The hosted area and database form the final key element of the middleware. As mentioned above, the hosted area and database were provided by Liverpool John Moores University. Without the hosted area, the Smart Serious Game and the portal counterparts could not have materialised. The Smart Serious Game requires players to act in the physical environment to progress, causing the game to require portability from a networking perspective. Therefore, a hosted area allows a user to $\log$ in and obtain their data from any location that has Internet connectivity. Furthermore, the production of web portals for academics and students that do not interact with the game requires a web-hosted area. The same criteria affect the decision to utilise a hosted database.

\subsection{Interactive Content}

SEA presents students with an avatar that reacts to the weekly measure of student academic engagement. The primary directive of the game is to maintain a happy avatar by obtaining a high score. In detail, there are five methods of progression in SEA: avatar happiness, avatar customisation, level mechanism, leaderboard, and achievements. The inclusion of the game scoring rule and completing achievements adheres to the theory of flow [33]. In-game objectives are challenging but achievable, to prevent students from disengaging from the game. Avatars and their related game mechanics were included based on research by Doyle et al. [34], who discovered players engage better with a game or serious game when they are represented as an avatar rather than an image of themselves. Finally, leaderboards were included based on research by Hanus and Fox [35] into gamification for improving Student Engagement. Hanus and Fox outlined the use of achievements and leaderboard as commonly utilised game mechanics in gamification for education.

The points required for each avatar look were determined by calculating the highest possible score a student could achieve during experiments, to ensure all looks could be unlocked. One thousand points are available for students to achieve during the first semester, accounting for the week away from campus due to Reading Week. All avatar customisation can be unlocked with eighthundred points, providing the best-achieving students with a margin of two hundred points. Though there are a lot if similarities between gamification and 


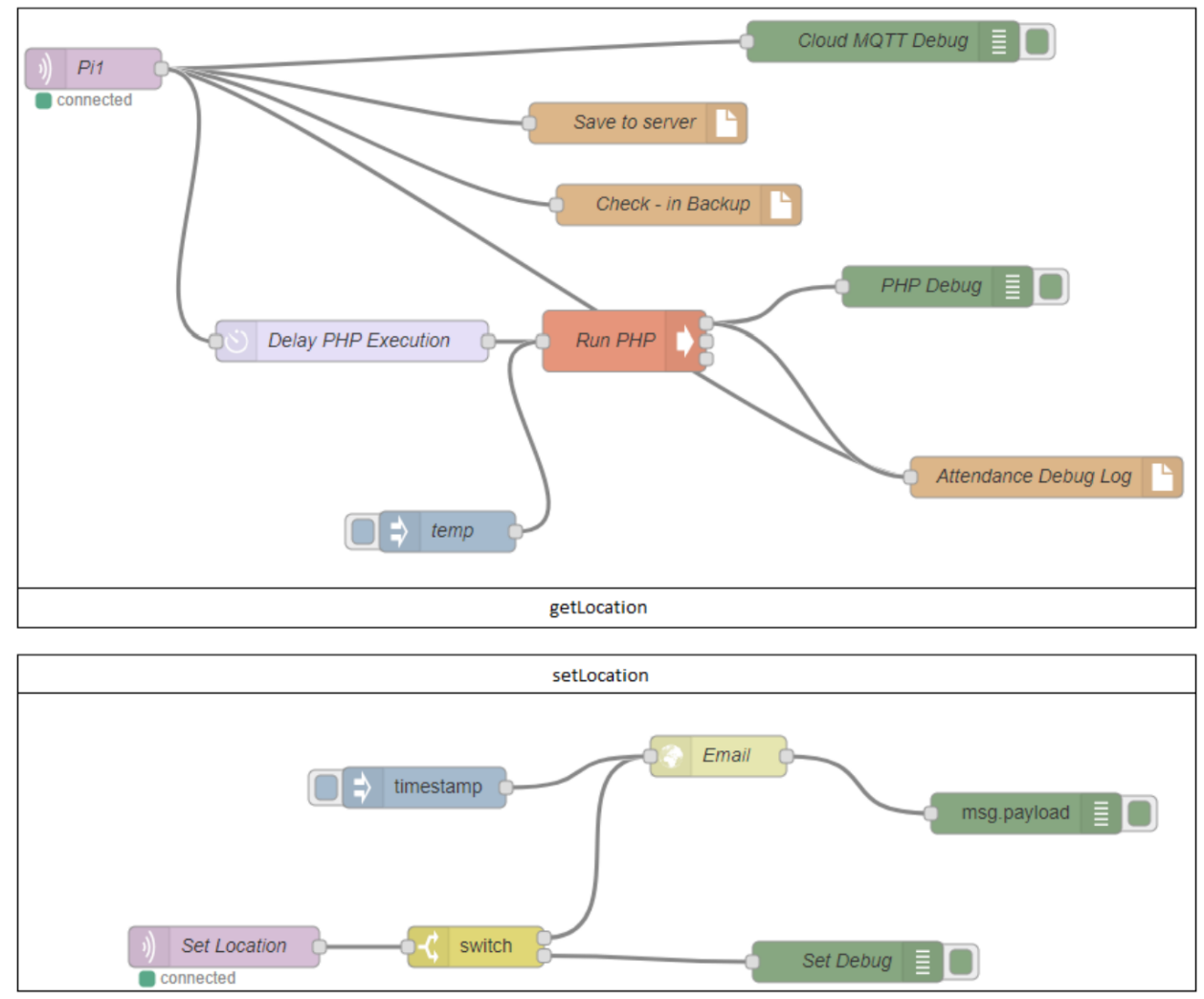

Figure 4: Node-RED visual script of the Internet of Things attendance system.

the developed content, SEA is a serious game that is played by actions in the physical world, where the aim of the game is to keep your avatar happy. SEA could be consider gamification in accordance to the state of the art definitions $[36,37]$ if there was no virtual avatar to maintain.

SEA includes a global leaderboard to add an element of competitiveness that is accessible in-game but hosted in a webpage. Students climb the leaderboard by performing well in the weekly measures of engagement. As the measure is a percentage, students can achieve a maximum of one hundred points a week towards the leaderboard. Weekly engagement scores are summed through the weeks of experiments, rewarding consistency in engagement. Furthermore, SEA rewards consistency through in-game achievements.

\section{Experiments and Findings}

The experiments aim to prove the validity of measuring student engagement using Serious Games and IoT, in turn proving the effectiveness of the proposed software framework, by utilising an $\mathrm{RCT}$, as a literature survey into 
Serious Games discovered a lack of RCTs when investigating the positive effects of Serious Games [38]. We hypothesize that an effective measure of student engagement through SEA validates the proposed framework used to develop the application. The effectiveness of the measure must match that of conventional measures of engagement. The first stage of the experiment process allocated participants to a group at random. The control group (Group A) gathered a measure of Student Engagement using self-reflection tools. The questionnaire required weekly completion. Students who failed to complete the survey received no mark for the week. According to existing literature, Student Engagement is a product of three core elements, Behavioural Engagement, Cognitive Engagement and Emotional Engagement. Behavioural Engagement drives other engagement elements and therefore becomes crucial to any measure of engagement $[10,30,31,9,32]$.

The treatment group (Group B), measured Student Engagement through SEA, which embeds self-reflection tools within the game. SEA accounts for attendance and punctuality values obtained from a purpose-built wireless sensor network, consisting of Raspberry Pi. An exact match in measures proves the validity of the software framework, whereas an improvement highlights a new approach for measuring Student Engagement. SEA achieves the measure of Engagement through a data algorithm that balances the number of classes a student has with the embedded self-reflection tools and the measures of behavioural Engagement obtained from the intrinsic sensor network, as seen in the equation below.

$$
E n=\left[\left(\left(\frac{S}{H e}\right) 100\right)\right]
$$

Elaborating on the above equation, En represents the measure of engagement. $E n$ is calculated by dividing the sum $S$ of weekly attendance, punctuality, and questionnaire scores, by the highest weekly engagement score possible $\mathrm{He}$. As the value of $E n$ represents a percentage, we must multiply by 100 to obtain a number that can be utilised for a game scoring system. Henry et al [6] provide further information on equation 1.

Partaking student's academic performance (grades), were analysed against retention in their programme, to validate the results of both groups (A \& B). All data regarding programme retention and academic performance were identified by the appropriate gatekeeper and remained anonymous to prevent participant identification. At the end of the experiment period, participants were required to fill out a questionnaire, sourcing their views of their experience during the project. The participants' personal opinions on their experience aided to evaluate the use of the IoT with Serious Games.

Undergraduate students from the Department of Computer Science at Liverpool John Moores University formed the participants of this experiment with the ethical approval number of $17 / \mathrm{CMS} / 003$. The recruitment campaign gathered 24 participants; however, two participants withdrew after signing up, resulting in twenty-two participants $(n=22)$ that completed the experiment. Existing 
research utilises similar sample sizes for control and treatment groups, such as investigating effects and experiences of Serious Games on undergraduate players [39], investigating the use of simulation games for vocabulary learning [40], and evaluating virtual worlds in regards to social, behavioural elements [41]. Based on these publications, this paper can form conclusions with the number of recruited students.

Participants had a computing background and covered all three levels of undergraduate study. Both groups interact with a computer-based application, one developed for the Web and the other for smartphones; therefore, no group nor participant gain any form of advantage or disadvantage based on their academic background.

\subsection{Response Rate}

The control and treatment groups utilised self-reflection tools. Group A sourced all its value from self-reflection, emulating conventional measures of Student Engagement, whereas Group B used a hybrid measure of self-reflection and data algorithm-generated score. Completing the self-reflection instrument was a core element of the Student Engagement measure for both groups. Group A completed the self-reflection instrument on average of $49.25 \%$ throughout the experiment. Their completion rates resulted in a standard deviation of 23.75 , highlighting a wide range of relative data points. Figure 5 illustrates the group's weekly completion percentage and the average engagement percentage $\mathrm{E}$. The completion rate in Group A fluctuated greatly, with minimum participation recorded in the first two weeks; however, the average engagement percentage recorded a downward trend overall. The data cannot conclusively state that low participation from a group will result in inflated measures of engagement but does state the inaccuracy in the measure that a limited amount of participation produces.

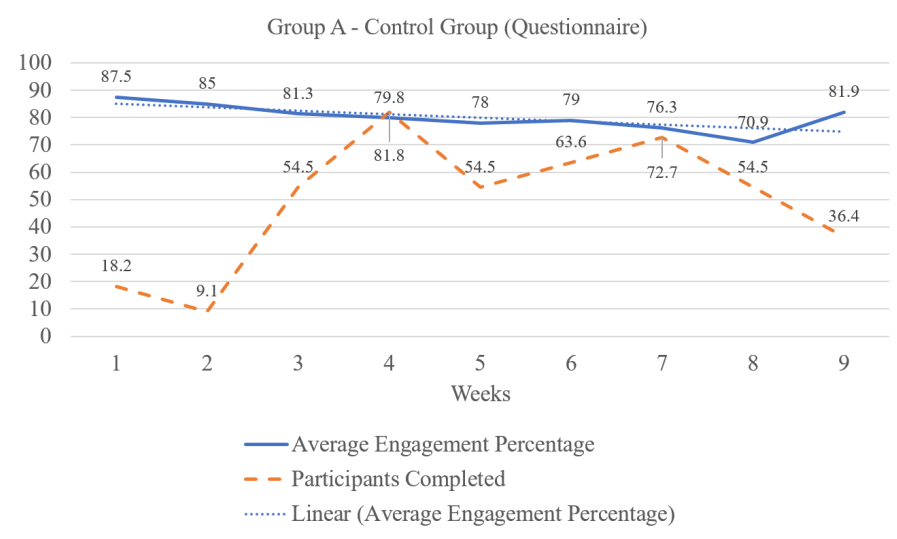

Figure 5: Group A (Control group): Weekly group average questionnaire completion and engagement 
Group B completed the self reflection instrument on average $80.05 \%$, an increase of $30.8 \%$ over the control group (Group A), producing a standard deviation value of 21.84, a decrease of 1.91 in comparison to Group A. Figure 6 displays the related findings. The lower standard deviation value highlights a consistently better response rate over Group A, a fact that the increase in average confirms, validating the results presented in the previous section.

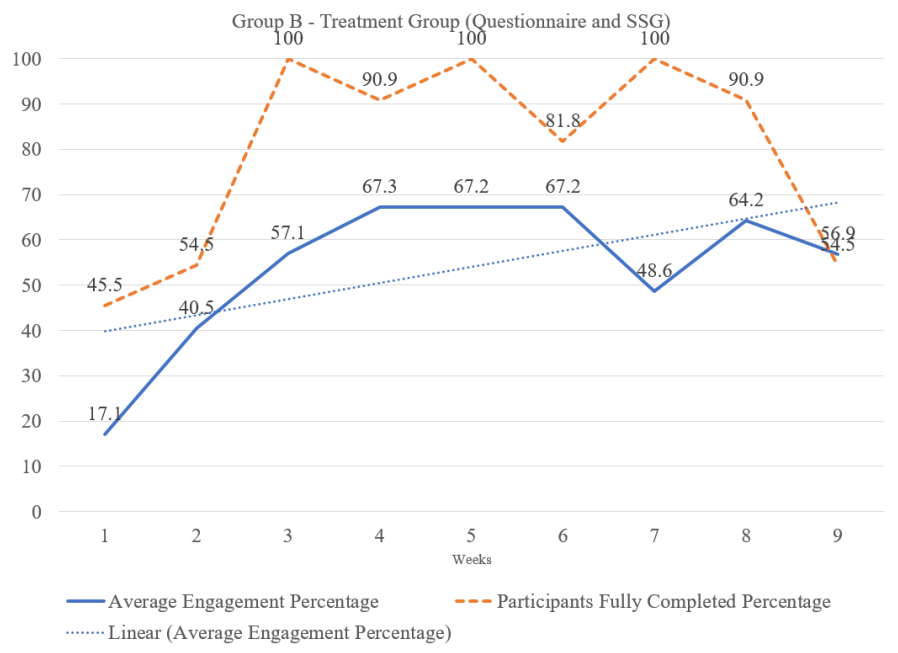

Figure 6: Group B (Treatment group): Weekly group average questionnaire completion and engagement

The stark difference between the participant experiences per group was the inclusion and exclusion of game immersion, respectively. Game immersion influences behaviour and performance in learning [42, 36, 43]. The recorded data suggests that game immersion positively affects completion rates with self-reflection instruments when embedded as a game mechanic. This finding can aid research across all domains that utilise questionnaires for obtaining data.

\subsection{Data Correlation}

The analysis into data correlation began by calculating the root mean average of engagement recorded through the control and treatment groups. Group A recorded a root mean of $80.1 \%$ whereas Group B recorded at $56.25 \%$, as Fig 7 illustrates. This result inclines that the students that did not interact with the game were far more engaged; however, we continued our data analysis to understand this result better.

The next objective is to calculate the relation between academic grade and measure of engagement. As a starting point, we calculated the mean average of each participant in each group and generating a total mean average for that group. We followed the same process for academic grades. Fig 8 illustrates the averages calculated for Group A. This group obtained an average engagement 


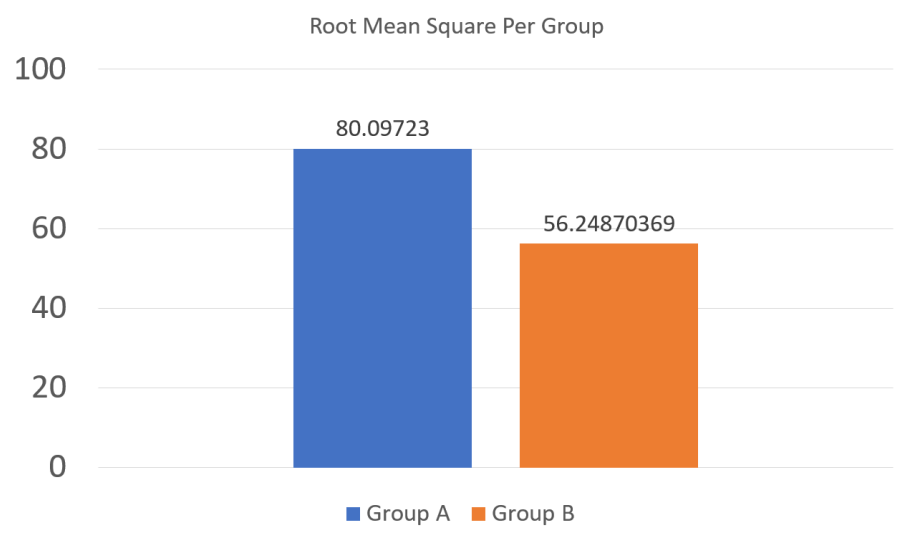

Figure 7: The calculated root mean of the control (Group A) and treatment (Group B) groups.

measure of $70.07 \%$ and an average grade of $61.14 \%$. Group B recorded an average engagement of $53.13 \%$ and an average academic grade of $63.72 \%$.

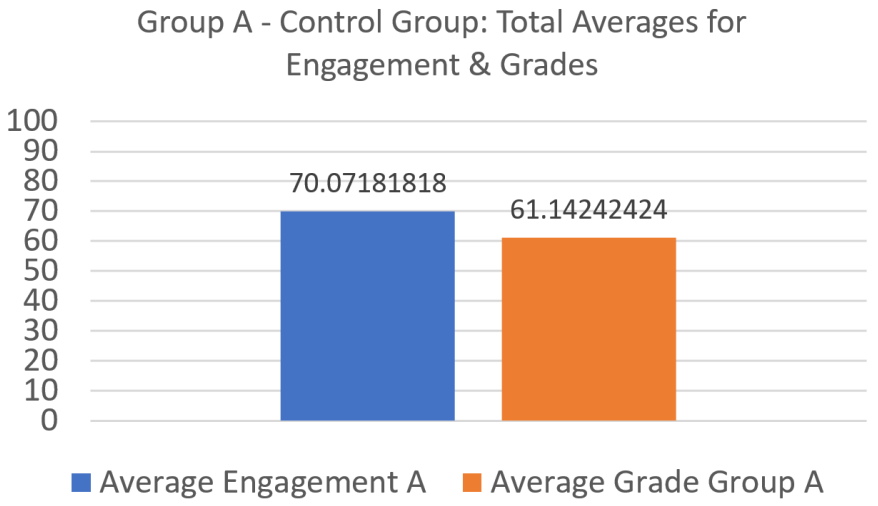

Figure 8: The average engagement against the average academic grade for the control group (Group A).

The averages from both groups suggested some form of correlation but this type of measure is not statistically validated. Therefore we continued the data analysis and utilised the correlation coefficient $\mathrm{R}$ to correlate between the student data points. The correlation coefficient R, also known as Pearson's $\mathrm{r}$ was presented in the 1880s [44] and is used to calculate the correlation between numerical arrays.

Fig 10 illustrates the averages on a per student basis for Group A. The orange line highlights engagement and the blue line shows the average grade.

In detail, the statistical algorithm produces the sum of two variables, formed by calculating the tendency of variance, divided by the degrees of freedom [45]. 


\section{Group B - Treatment Group: Total Averages for}

Engagement \& Grades

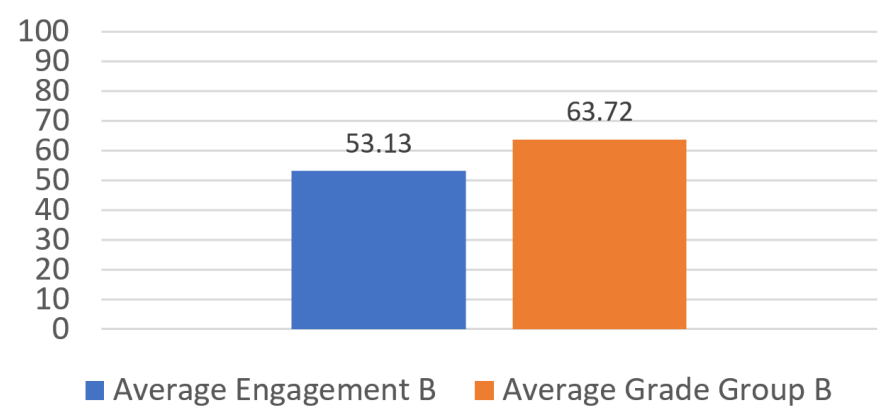

Figure 9: The average engagement against the average academic grade for the treatment group (Group B).

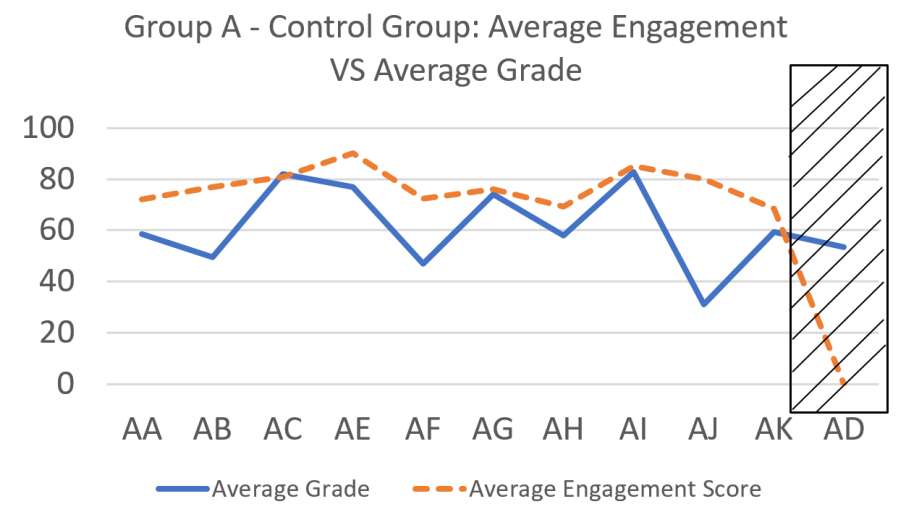

Figure 10: Comparison between grade and engagement score from conventional measure

The nature of this statistical algorithm allows this paper to investigate the correlation between the array of grades and an array of engagement scores in an attempt to validate the acquired measures of engagement.

Initially, the data from Group A produced a correlation coefficient R-value of 0.27 , indicating a slight upward correlation. Statistically, a value of 0.27 would not indicate a correlation between the measure of engagement from selfreflection and academic grades. The further investigation highlighted a single student, code-named AD to protect anonymity, had approached the respective programme leader of the course, expressing his desire to withdraw from the university. As no data points were captured from the participant, the academic grade was classified as noise. Emitting data noise, the R-value increased to 0.44 . This result presents a weak upward correlation for the group. The probability value $p$ did not produce statistical significance for this correlation $r$. 
Figure 11 presents the data per student for Group B. The data presented produced an initial correlation coefficient $\mathrm{R}$ of 0.22 , illustrating no real correlation between the Student Engagement measure and academic performance. Hardware issues became apparent after interviewing the students that produced a wide margin between values. Both students stated that their Bluetooth would not register their attendance even though they were present in classes. They mentioned this caused frustration and a tendency to disengage from the experiment. After verifying this claim by analysing the data, students $B J$ and $B H$ were classified as noise due to hardware issues. Other students with the same phone did not reproduce the same issues, removing the possibility of a compatibility error due to the phone model and make.

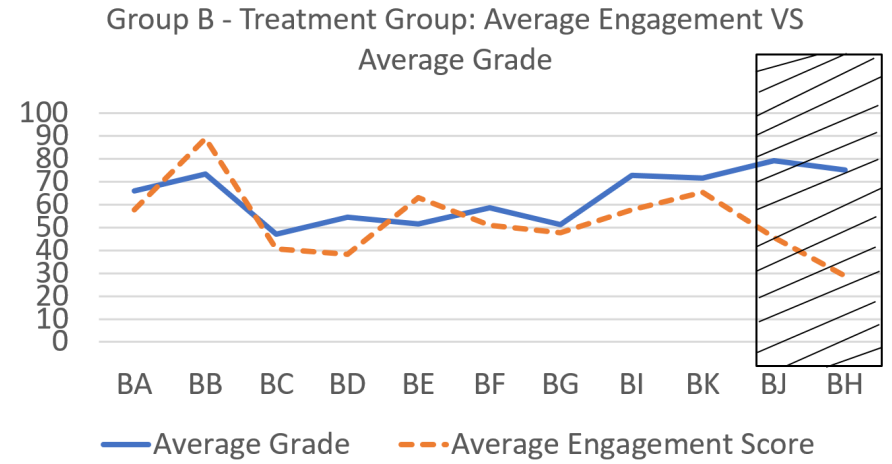

Figure 11: Comparison between grade and engagement score from SSG measure

After defining and removing data noise in the group, the correlation coefficient $\mathrm{R}$ improved to 0.71 , presenting a strong upward correlation. The statistical significance $p$ measures the probability of a result reoccurring based on the level of random sample [46], and produced a value of .032114, providing significant at $p<.05$, therefore validating the measure of engagement and the effectiveness of the software framework. Though the average measures of engagement and the respective academic performance per group were similar in both groups, when analysing each student's measure in comparison to their academic performance, the data provides a new perspective that displays data noise and a great difference in correlation between the groups.

\subsection{Data Evaluation}

Data evaluation utilised data obtained from the experiments with data obtained from an interview questionnaire provided to participants at the end, to study the shared views of students against the data findings discussed in the previous sections. The interview questionnaire included open-ended questions for sourcing the qualitative responses of participants to better understand the quantitative data detailed previously. 
Out of the total participants in both groups (n=22), 14 participants responded. Participants that presented abnormal data patterns were pursued to complete the questionnaire for understanding if their data was noise. From the respondents, six belong to Group A, with the remaining eight belonging to Group B. Question 1 investigates the effect of the experiment on Student Engagement, according to the participant's belief. From Group B, $75 \%$ felt there was a direct effect on their engagement. The remaining two participants stated no impact. Though the majority that responded from this group believed there was an increase in engagement, there is no statistical proof of improvement from the quantitative data. Within Group A, 2 out of 6 felt an improvement in their engagement. SEA did not set out to improve student engagement and as such these results do not mitigate the effectiveness of the application. SEA aims to provide a new method of measuring student engagement, allowing future research to promote behavioural change through the game.

Question 2 sources the level of interaction. Group B responded with phrases such as "I interacted with it as I attended university each day" and "I interacted with the game in every class I attended, I liked to gather the points and see how high I could get on the leader-board", highlighting the level of participation from the group and the reflecting the measure of engagement. Group A responded with phrases such as 7 or 9 times throughout the semester didn't interact due to coursework or I did not, I was in the group which did not use the game. Several students responded with I interacted with as many chances as I got. Their responses reflect the higher variance presented in the group and the overall reduction in participation and the effect of game immersion over conventional measures of engagement. We highlight the self-awareness of students with their participation levels with the experiment.

Question 3 gathers the level of comfort students had with the technology they used as part of the experiment. Students from both groups unanimously stated there was no discomfort with the technology used. The result removes technology interaction as a factor of consideration when measuring student engagement.

Question 4 follows up on the use of technology but questions the usability for the web application and Serious Game, respectively. Both groups rated usability as "good" and found the respective software easy to use and understand, supporting the responses to the previous question.

Question 5 focuses on critical software bugs that could cause data noise in either group. Two participants from Group B reported issues with Bluetooth and obtaining an attendance mark even though they were present in lectures and practical sessions. The two students also presented a high variance between their Student Engagement measure and their academic performance. Their data was defined as noise, after investigating the data further and discovering the two students experienced technical difficulties that hindered their attendance and punctuality measures.

Finally, Question 6 collects participant views on the overall experience with the experiment. Most participants from both groups chose not to add any further comments regarding any positive or negative experiences. Two students 
did suggest improving the data capture and data algorithm by accounting for how long students remained in a session, rather than attendance alone.

\section{Conclusion and Future Work}

Although the recorded data did not produce a significant difference in Student Engagement $E$ between control and treatment group, we validated the measure of engagement through an SSG named SEA, that combines Serious Games with IoT. The aim of the research project was producing a measure of student engagement using Serious Games and the Internet of Things. The lack of significant difference in Student Engagement $E$ evidences the new proposed method of measuring student engagement is as valid as conventional measures. We validated a software framework for combining Serious Games and IoT that is not bespoke to SEA, bridging a gap in the existing literature. The recorded data highlighted a benefit in participation during experiments through game immersion, supporting the development of similar projects in future and the utilisation of computer algorithms for calculating engagement through a game. The presented software framework supports the future growth of data-driven games that utilise the IoT to present information in game-play within the vir-

tual environment based on factors that are collected through the IoT from the physical environment.

There are several future directions of research originating from this paper. New environmental data combined with the presented framework opens the potential for investigating the effects of environmental events on behavioural change, such as motivation, as the presented framework is application neutral and it is ideal for instantiating this type of research. Furthermore, we propose the inclusion of time spent in class as a variable to be processed by the data algorithm, that investigates a potential improvement in Student Engagement measure, as a future direction.

\section{References}

[1] L. Atzori, A. Iera, G. Morabito, The Internet of Things: A survey, Computer Networks 54 (2010) 2787-2805.

[2] Z. Khalid, N. Fisal, M. Rozaini, A Survey of Middleware for Sensor and Network Virtualization, Sensors 14 (2014) 24046-24097.

[3] D. R. Michael, S. L. Chen, Serious Games: Games That Educate, Train, and Inform, volume October 31, 2005.

[4] S. Tang, M. Hanneghan, C. Carter, A Platform Independent Game Technology Model for Model Driven Serious Games Development., Electronic Journal of e-Learning 11 (2013) 61-79.

[5] D. Sharma, J. C. Lakhmi, M. Favorskaya, R. J. Howlett, Fusion of Smart , Multimedia and Computer Gaming Technologies, 1 ed., Springer International Publishing, 2015. 
[6] J. Henry, S. Tang, M. Hanneghan, C. Carter, A framework for the integration of serious games and the internet of things (iot), in: 2018 IEEE 6th International Conference on Serious Games and Applications for Health (SeGAH), IEEE, 2018, pp. 1-8.

[7] B. L. Rich, J. A. Lepine, E. R. Crawford, Job engagement: Antecedents and effects on job performance, Academy of Management Journal 53 (2010) $617-635$.

[8] A. J., Determinants of employee engagement and their impact on employee performance, International Journal of Productivity and Performance Management 63 (2014) 308-323.

[9] E. A. Skinner, M. J. Belmont, Motivation in the classroom: Reciprocal effects of teacher behavior and student engagement across the school year., Journal of Educational Psychology 85 (1993) 571-581.

[10] J. J. Appleton, S. L. Christenson, D. Kim, A. L. Reschly, Measuring cognitive and psychological engagement: Validation of the Student Engagement Instrument, Journal of School Psychology 44 (2006) 427-445.

[11] J. D. Finn, Withdrawing from School, Review of Educational Research 59 (1989) 117-142.

[12] F. Bonomi, R. Milito, J. Zhu, S. Addepalli, Fog computing and its role in the internet of things, in: Proceedings of the first edition of the MCC workshop on Mobile cloud computing - MCC '12, ACM Press, New York, New York, USA, 2012, p. 13.

[13] J. Lin, W. Yu, N. Zhang, X. Yang, H. Zhang, W. Zhao, A survey on internet of things: Architecture, enabling technologies, security and privacy, and applications, IEEE Internet of Things Journal 4 (2017) 1125-1142.

[14] A. Gilchrist, Industry 4.0: the industrial internet of things, Apress, 2016.

[15] E. I. Konstantinidis, The interplay between IoT and serious games towards personalised healthcare, 9th International Conference on Virtual Worlds and Games for Serious Applications (2017) 249-252.

[16] C. Garcia-Garcia, F. Terroso-Saenz, F. Gonzalez-Burgos, A. F. Skarmeta, Integration of serious games and IoT data management platforms to motivate behavioural change for energy efficient lifestyles, GIoTS 2017 - Global Internet of Things Summit, Proceedings (2017).

[17] H.-y. Kim, A design and implementation of a framework for games in IoT, The Journal of Supercomputing (2017).

[18] E. Lotfi, B. Mohammed, Players Performances Analysis based on Educational Data Mining Case of Study : Interactive Waste Sorting Serious Game, International Journal of Computer Applications 108 (2014) 13-18. 
[19] L. Elaachak, A. Belahbibe, M. Bouhorma, Towards a System of Guidance, Assistance and Learning Analytics Based on Multi Agent System Applied on Serious Games, International Journal of Electrical and Computer Engineering (IJECE) 5 (2015) 344-354.

[20] A. Zanella, N. Bui, A. Castellani, L. Vangelista, M. Zorzi, Internet of things for smart cities, IEEE Internet of Things Journal 1 (2014) 22-32.

[21] S. Vicini, S. Bellini, A. Rosi, A. Sanna, An internet of things enabled interactive totem for children in a living lab setting, in: 2012 18th International ICE Conference on Engineering, Technology and Innovation, IEEE, 2012, pp. $1-10$.

[22] K. Heljakka, P. Ihamäki, Preschoolers learning with the internet of toys: From toy-based edutainment to transmedia literacy, in: Seminar. net, volume 14, 2018, pp. 85-102.

[23] Y.-N. Lien, Mobile edutainment system-national palace museum as an example, in: 2015 3rd International Conference on Future Internet of Things and Cloud, IEEE, 2015, pp. 597-601.

[24] M. Kranz, P. Holleis, A. Schmidt, Embedded interaction: Interacting with the internet of things, IEEE internet computing (2009) 46-53.

[25] M. Pouryazdan, C. Fiandrino, B. Kantarci, T. Soyata, D. Kliazovich, P. Bouvry, Intelligent gaming for mobile crowd-sensing participants to acquire trustworthy big data in the internet of things, IEEE Access 5 (2017) 22209-22223.

[26] D. N. Crowley, J. G. Breslin, P. Corcoran, K. Young, Gamification of citizen sensing through mobile social reporting, 4th International IEEE Consumer Electronic Society - Games Innovation Conference, IGiC 2012 (2012).

[27] S. R. Islam, D. Kwak, M. H. Kabir, M. Hossain, K.-S. Kwak, The internet of things for health care: a comprehensive survey, IEEE Access 3 (2015) 678-708.

[28] M. Swan, Sensor mania! the internet of things, wearable computing, objective metrics, and the quantified self 2.0, Journal of Sensor and Actuator networks 1 (2012) 217-253.

[29] P. Yang, Prls-inves: A general experimental investigation strategy for high accuracy and precision in passive rfid location systems, IEEE Internet of Things Journal 2 (2014) 159-167.

[30] B. D. Coller, D. J. Shernoff, Video Game-Based Education in Mechanical Engineering: A Look at Student Engagement, International Journal Of Engineering Education 25 (2009) 308-317. 
[31] M. M. Handlesman, W. Briggs, N. Sullivan, A. Towler, A measure of college student course engagement., The Journal of Educational Research 98 (2005) 184-192.

[32] J. H. Brockmyer, C. M. Fox, K. A. Curtiss, E. Mcbroom, K. M. Burkhart, J. N. Pidruzny, Journal of Experimental Social Psychology The development of the Game Engagement Questionnaire : A measure of engagement in video game-playing, Journal of Experimental Social Psychology 45 (2009) 624-634.

[33] M. Csikszentmihalyi, Flow: The Psychology of Optimal Experience., Academy of Management Review 16 (1991) 636-640.

[34] J. Doyle, C. Bailey, B. Dromey, C. N. Scanaill, BASE - An interactive technology solution to deliver balance and strength exercises to older adults, International Conference on Pervasive Computing Technologies for Healthcare (PervasiveHealth) (2010) 1-5.

[35] M. D. Hanus, J. Fox, Assessing the effects of gamification in the classroom: A longitudinal study on intrinsic motivation, social comparison, satisfaction, effort, and academic performance, Computers and Education 80 (2015) 152-161.

[36] S. Deterding, D. Dixon, R. Khaled, L. Nacke, From game design elements to gamefulness: Defining gamification, Proceedings of the 15th International Academic MindTrek Conference on Envisioning Future Media Environments - MindTrek '11 (2011) 9-11.

[37] F. Groh, Gamification: State of the art definition and utilization, Institute of Media Informatics Ulm University 39 (2012) 31.

[38] T. M. Connolly, E. Boyle, T. Hainey, J. M. Boyle, A systematic literature review of empirical evidence on computer games and serious games, Computers and Education 59 (2010) 661-686.

[39] Y. T. Chiang, C. Y. Cheng, S. S. Lin, The effects of digital games on undergraduate players' flow experiences and affect, in: Proceedings - 2nd IEEE International Conference on Digital Game and Intelligent Toy Enhanced Learning, DIGITEL 2008, 2008, pp. 157-159.

[40] M. Miller, V. Hegelheimer, The SIMs meet ESL Incorporating authentic computer simulation games into the language classroom, Interactive Technology and Smart Education 3 (2006) 311-328.

[41] A. De Lucia, R. Francese, I. Passero, G. Tortora, Development and evaluation of a virtual campus on Second Life: The case of SecondDMI, Computers \& Education 52 (2009) 220-233. 
[42] I. Blohm, J. M. Leimeister, Gamification: Design of IT-based enhancing services for motivational support and behavioral change, Business and Information Systems Engineering 5 (2013) 275-278.

[43] R. Garris, R. Ahlers, J. E. Driskell, Games, Motivation, and Learning: A Research and Practice Model, Simulation \& Gaming 33 (2002) 441-467.

[44] S. M. Stigler, Francis Galton's Account of the Invention of Correlation, Statistical Science 4 (1989) 73-79.

[45] S. Boslaugh, P. Watters, Statistics in a Nutshell: A Desktop Quick Reference, 2008.

[46] R. L. Wasserstein, N. A. Lazar, et al., The asa's statement on p-values: context, process, and purpose, The American Statistician 70 (2016) 129133. 